Revista de Economia Política, vol. 32, $n^{o} 3$ (128), pp. 459-478, julho-setembro/2012

\title{
Mudança estrutural e mercado de trabalho no Brasil
}

\author{
RAFAEL CAMARGO DE PAULI \\ LUCIANO NAKABASHI \\ ARMANDO VAZ SAMPAIO*
}

\begin{abstract}
Structural changes and labor market in Brazil. In the present article, we attempt to identify the sources of the changes in the labor schooling level in the three main sectors of the Brazilian economy: manufacturing, services and agriculture. It was verified that, despite the changes in the product and employment among sectors, mainly in the 1990s, the relative demands for qualified workers has not experimented significant changes. Moreover, in the periods in which schooling has increased more, the workers' wage has decreased more. This fact suggests that the increase in labor qualification was mainly due to the increase of this factor supply. The structural changes had contributed, in general, in a marginal and negative way to labor force level of qualification demand in all the three sectors.

Keywords: labor schooling; structural changes; demand for qualified labor; supply of qualified labor.

JEL Classification: F16; I20; J22; J23; J24.
\end{abstract}

\section{INTRODUCÃO}

A economia brasileira passou por importantes alterações macroeconômicas a partir dos anos 1980. Para citar algumas, temos o processo de abertura econômica no Brasil que se intensificou a partir do final da década de 1980 e, principalmente, no início dos anos 1990. Outro fato de grande relevância foi o fim do processo

\footnotetext{
* Analista Econômico da COHAB, e-mail:rafaelcdp@gmail.com; Professor Doutor da FEA-RP/USP e pesquisador do CNPQ, e-mail luciano.nakabashi@gmail.com; e Professor Adjunto do Departamento de Economia da UFPR, e-mail: avsampaio@ufpr.br. Submetido: 13/janeiro/2011; Aprovado: 31/agosto/2011.
} 
inflacionário, em 1994, além das mudanças na política cambial e de juros, nos fluxos de capitais etc. Alguns autores que analisaram e destacaram a importância dessas variações na estrutura e no dinamismo da economia brasileira são Salm, Saboia e Carvalho (1997), Bonelli e Fonseca (1998), Carvalho e Feijó (2000), Carvalheiro (2003), Ferraz, Kupfer e Iootty (2004), Feijó Carvalho e Almeida (2005) e Nassif (2006).

Com a ocorrência de mudanças significativas na estrutura produtiva da economia brasileira, também seria de esperar alterações relevantes no mercado de trabalho. Uma das modificações relevantes pelo qual o mercado de trabalho atravessou foi o ganho de participação do setor de serviços na mão de obra empregada (e no produto) em relação aos demais setores da economia (Cruz et al., 2007). Esse tipo de mudança estrutural é natural no decorrer do desenvolvimento econômico, como enfatizado por Rowthorn (1999) e Rowthorn e Ramaswamy (1999), mas variáveis como abertura comercial e apreciação cambial podem acelerar esse processo. Um aspecto importante dessas mudanças estruturais são os seus efeitos na demanda por qualificação de mão de obra ${ }^{1}$.

Por outro lado, ocorreram mudanças significativas na qualificação da mão de obra pelo aumento de sua oferta, considerando o mesmo período de tempo. Presenciamos, a partir do final da década de 1980, um aumento expressivo no nível de escolarização dos trabalhadores brasileiros devido à implantação de programas governamentais com esse objetivo. Entretanto, as evidências empíricas apresentadas nesse estudo mostram que a melhora na qualificação da mão de obra não têm sido aproveitadas em toda sua potencialidade. Isso vem ocorrendo porque a demanda por esse fator não acompanhou a expansão de sua oferta e/ou porque a elevação na qualificação se deu em termos meramente quantitativos, ou seja, sem impactos relevantes no nível de habilidades dos trabalhadores.

Tendo em mente os fenômenos apresentados acima, o objetivo do artigo é verificar como as transformações estruturais observadas na economia brasileira influenciaram a demanda por trabalhadores segundo seus níveis de qualificação e de acordo como os diferentes setores da economia, além de verificar qual a sua interação com a oferta de trabalho. Nesse sentido, a contribuição do presente artigo em relação à literatura é a realização dessa análise através de gráficos e do método de decomposição (shift-share) com os dados da RAIS, no período 1985-2007. Ressaltamos ainda que não temos conhecimento de estudos que tratam de forma mais detalhada a relação entre mudança estrutural e mercado de trabalho para o Brasil com essa metodologia.

Os resultados mostram que, de forma geral, a demanda por trabalhadores qualificados não aumentou nos períodos em que ocorreram os maiores ganhos de produtividade em termos relativos ou absolutos, na indústria de transformação e no setor de serviços. Por outro lado, ocorreram aumentos expressivos nas quanti-

\footnotetext{
${ }^{1}$ No presente artigo, utilizamos os termos qualificação da mão de obra e escolaridade da mão de obra como intercambiáveis.
} 
dades de trabalhadores com qualificação intermediária (níveis de ensino fundamental e médio completos) em todos os setores. Entretanto, os salários dos mesmos caíram de forma acentuada no período de maior expansão da oferta de trabalhadores com esse nível de qualificação, indicando que aumentos da escolaridade média dos trabalhadores brasileiros responderam, em maior medida, à expansão da oferta de ensino no país. Além disso, as mudanças estruturais ocorridas, principalmente na década de 1990, não têm agido de modo a aumentar a demanda por esse contingente crescente de trabalhadores com maior qualificação. Por fim, os aumentos de produtividade observados na década de 1990 não foram acompanhados por aumentos na quantidade de trabalhadores qualificados nos serviços e na indústria de transformação.

O trabalho está dividido em quatro seções além desta introdução e das conclusões. Na próxima seção, apresentamos uma breve revisão de trabalhos empíricos sobre o processo de mudança estrutural pelo qual passam os países, além de alguns estudos que enfatizam os efeitos da abertura comercial sobre o mercado de trabalho. Na terceira seção, iniciamos a análise empírica a partir da verificação do comportamento do produto, do emprego e da produtividade nos setores da economia brasileira. Finalmente, na quarta seção, realizamos uma análise da evolução da escolaridade dos trabalhadores nos diferentes setores, comparando os resultados obtidos com aqueles da seção anterior.

\section{MUDANÇA ESTRUTURAL E O MERCADO DE TRABALHO}

Mudanças estruturais são variáveis-chave para se entender as variações na demanda por trabalho e, portanto, a dinâmica desse mercado. Elas também são bastante comuns, sendo amplamente estudadas por diversos analistas. Por exemplo, Krüger (2008) verificou que, entre 1948 e 2000, a participação do setor secundário no valor agregado caiu de $30 \%$ para $18 \%$, na economia americana. A participação do setor de serviços passou de $45 \%$ para $68 \%$.

Em uma compilação com dados de Maddison (1995) e do World Development Indicators (2004), Sasaki (2007) encontrou resultados parecidos para França e Reino Unido, no período 1950-2001². Na mesma linha, Palma (2005) mostrou que, em média, entre 1960 e 1998, os países desenvolvidos apresentaram uma queda da proporção do emprego industrial de $26,5 \%$ para $17,3 \%$. Em relação aos países em desenvolvimento, o autor verificou a existência de uma queda na participação do emprego industrial nos países do cone sul e Brasil de 17,4\%, em 1960, para 11,8\%, em 1998.

Considerando a economia brasileira, Feijó Carvalho e Almeida (2005) argu-

\footnotetext{
${ }^{2}$ O primeiro país apresentou uma queda de $35 \%$ para $24 \%$ da participação da manufatura no emprego total e aumento de $37 \%$ para $74 \%$ da participação do setor de serviços. No Reino Unido, os valores passaram de $45 \%$ para $25 \%$ e de $50 \%$ para $73 \%$, respectivamente.
} 
mentam que a queda do setor industrial, notadamente a indústria de transformação, deve-se à política macroeconômica adotada a partir de 1990 e, principalmente, à política cambial e de taxa de juros entre 1994 e 1999. Em relação aos serviços, este absorve a mão de obra liberada da indústria, como apontado por Cruz et al. $(2007)^{3}$.

Em relação à produtividade da indústria brasileira Salm, Saboia e Carvalho (1997), Bonelli e Fonseca (1998), Carvalho e Feijó (2000), Carvalheiro (2003), Ferraz, Kupfer e Iootty (2004) e Nassif (2006) verificam incrementos a partir da década de 1990. Não há consenso quanto às suas causas, mas as explicações apontam para a importância da maior abertura econômica no período. Bonelli e Fonseca (1998) enfatizam as mudanças organizacionais e a incorporação "sem precedentes" de métodos de gestão administrativa nas empresas industriais instaladas no Brasil. Ferraz, Kupfer e Iootty (2004) ressaltam ainda a importância da introdução de tecnologias de informação e equipamentos na produção e administração das empresas. Salm, Saboia e Carvalho (1997, p. 394) concluem que:

houve efetivamente um forte crescimento da produtividade [do trabalho industrial] na primeira metade dos anos 1990 [...] associado à introdução de um conjunto amplo de métodos de gestão da produção, voltados para o aumento da competitividade das empresas. Isto explicaria a convivência de altas taxas de crescimento da produtividade com baixas taxas de investimento em capital físico.

Em geral, pode-se dizer que a maior abertura econômica observada no período possibilitou, por um lado, a modernização de firmas e, por outro, expulsou do mercado as empresas menos produtivas. Esses resultados sobre a produtividade mostram que seria de esperar uma maior demanda por mão de obra qualificada no setor industrial brasileiro a partir de 1990. Para isso, deve-se assumir que a maior produtividade tenha sido acompanhada de complementaridade entre capital e trabalho qualificado ou que os novos métodos gerenciais pressionaram o aumento da qualificação dos trabalhadores no setor industrial.

Neste sentido, Fernandes e Menezes-Filho (2002) analisaram a demanda e a oferta de trabalhadores qualificados, intermediários e não qualificados através da decomposição da evolução dos salários relativos. As conclusões principais do estudo apontam para um aumento da demanda por trabalhadores qualificados em tarefas complexas e da demanda por trabalhadores intermediários em tarefas simples. Machado e Moreira (2001) encontram evidências de que a abertura comercial

\footnotetext{
${ }^{3}$ Alguns estudos sugerem que esses setores (indústria e serviços) possuem dinâmicas distintas como, por exemplo, Cruz et al. (2007), Scatolin et al. (2007), Dasgupta e Singh (2006, 2005) Palma (2005), Feijó, Carvalho e Almeida (2005) e Bresser e Nakano (2003). Desse modo, essas mudanças trariam mudanças relevantes no dinamismo econômico e no mercado de trabalho.
} 
teve impactos negativos na demanda relativa de trabalho menos qualificado. Menezes-Filho e Rodrigues Jr. (2003), por sua vez, argumentam que a transferência de tecnologia é um dos principais fatores para explicar o aumento da demanda por mão de obra qualificada no Brasil. Ainda, Andrade e Menezes-Filho (2005) observam que parece estar ocorrendo uma elevação na demanda relativa por trabalhadores com qualificação intermediária inferior ao aumento da sua oferta relativa. Adicionalmente, os autores encontram que a demanda por trabalhadores com ensino superior completo não sofreu alterações expressivas.

\section{DADOS RECENTES DO BRASIL: PRODUTO, EMPREGO E PRODUTIVIDADE}

\section{Composição do produto e do emprego entre os setores privados da economia brasileira}

Uma das formas pela qual a mudança estrutural se configura é através da mudança da composição do produto da economia entre setores. Para verificar isso, apresentamos o Gráfico 1 com a participação dos três setores principais da economia.

Gráfico 1: Participação dos setores no produto interno bruto - 1990 - 2009

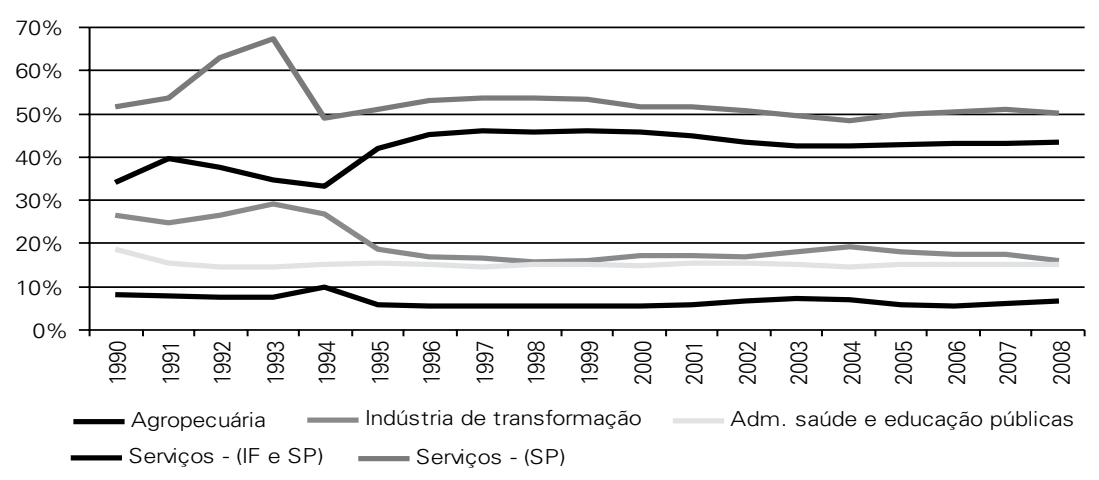

Fonte: Elaboração própria com dados das Contas Nacionais/IBGE, tabelas 1846 e 1620 do SIDRA. Nota: Serviços - (IF e SP): Serviços exceto intermediação financeira e administração, saúde e educação públicas. Serviços - (SP): Serviços exceto administração, saúde e educação públicas

O Gráfico 1 mostra que o setor de serviços, exceto o setor público e a intermediação financeira, apresentaram aumentos de participação, a partir de 1994. A indústria de transformação apresentou queda substancial entre 1993 e 1995, a partir do que se estabilizou. A agropecuária também apresentou tendência pouco volátil, o que revela a estabilidade da estrutura produtiva brasileira, no período, quando consideramos os setores em nível muito agregado.

Para verificar a evolução do emprego segundo setores da economia, foram utilizados dados de pessoas ocupadas das Contas Nacionais do IBGE, entre 1990 e 2006. O Gráfico 2 ilustra os resultados para a agropecuária; indústria de trans- 
formação; administração, saúde e educação públicas; serviços, exceto setor público e serviços exceto setor público e financeiro.

Gráfico 2: Participaçoes dos setores no emprego - 1990 - 2006

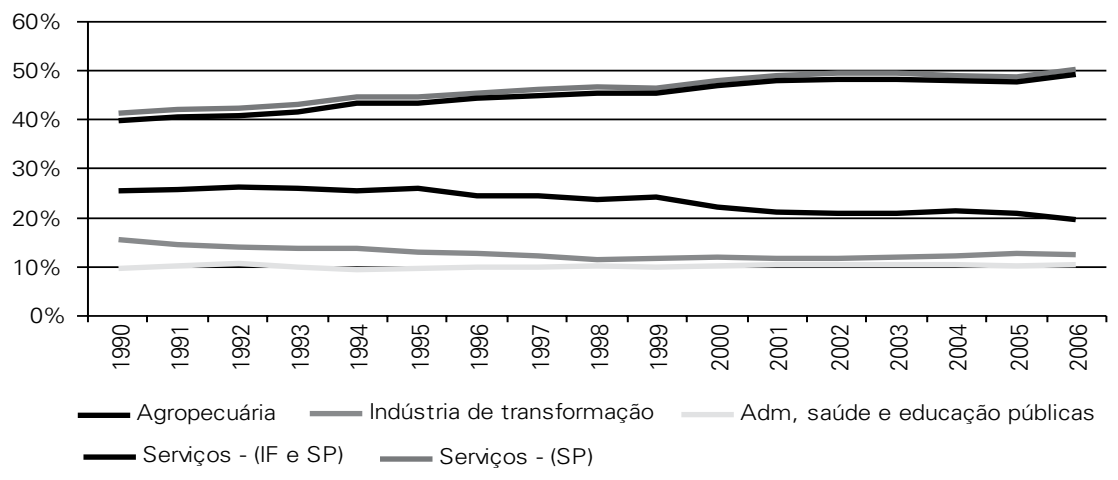

Fonte: Elaboração própria com dados das Contas Nacionais — IBGE.

Observa-se um claro aumento do emprego do setor serviços de cerca de 10 p.p., além de queda da participação do emprego da agropecuária e da indústria de transformação de cerca de 6 p.p. e 5 p.p., respectivamente. A redução da indústria de transformação, entretanto, ocorreu entre 1990 e 1998, a partir do que se estabilizou, voltando a subir ligeiramente, após 2003. Esses resultados mostram que ocorreram significativas alterações na estrutura do emprego mesmo em um nível muito agregado. Conjuntamente com os dados apresentados no Gráfico 1, podemos concluir que a produtividade é um elemento mais relevante para a indústria em relação ao setor de serviços. Em nossa análise empírica da decomposição essas mudanças são ainda mais notáveis na medida em que usamos um maior nível de desagregação.

\section{Produtividade da indústria de transformação e dos serviços no Brasil}

Os setores analisados nesta seção são a indústria de transformação e os serviços para ressaltar o padrão de produtividade nos dois principais setores da economia brasileira. A produtividade da indústria de transformação é apresentada no Gráfico 3. Foram utilizados os dados do Valor Adicionado (VA) e do Pessoal Ocupado (PO) do Sistema de Contas Nacionais (SCN) do IBGE, para o período 1990-2006. Esta base foi escolhida devido à maior uniformidade apresentada ao longo do período analisado 4 .

\footnotetext{
${ }^{4}$ Os VA de 1990 a 2007 foram calculados a partir da variação real anual do setor, tendo por base o valor adicionado do ano de 2008. Os anos de 2007 e 2008 não foram incluídos, pois ainda não há dados do pessoal ocupado. Os dados do PO da PNAD não foram utilizados, pois diferem substancialmente daqueles do SCN.
} 
Gráfico 3: Produtividade e variações percentuais acumuladas do pessoal ocupado e valor adicionado da indústria de transformação - 1990 - 2006

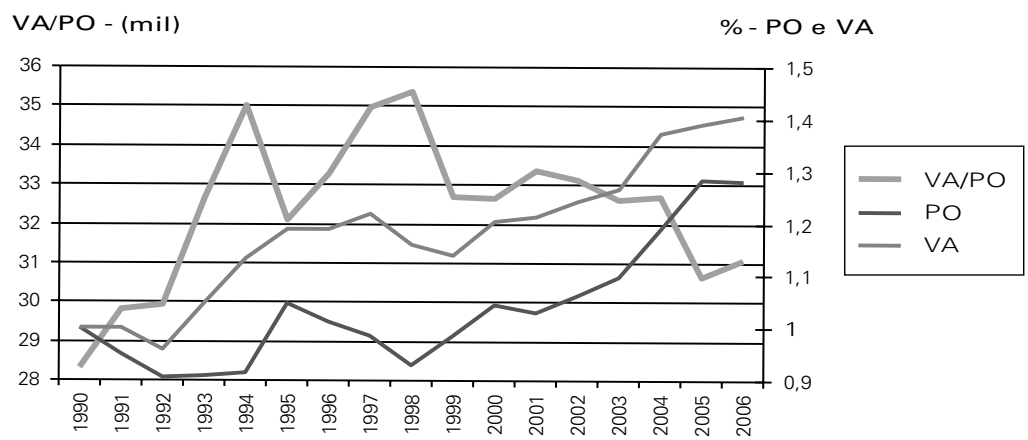

Fonte: Elaboração própria com dados do Sistema de Contas Nacionais — IBGE.

Observa-se que a produtividade do trabalho da indústria de transformação sofreu grandes oscilações, mas podemos destacar que ocorreu uma elevação até 1998, com posterior queda. A evolução inicial apresentada acima está de acordo com os dados da produtividade brasileira verificados por outros autores, como Rossi Jr. e Ferreira (1999), Carvalho e Feijó (2000), Carvalheiro (2003), e Ferraz, Kupfer e Iootty $(2004)^{5}$. Segundo os dados apresentados acima, a indústria de transformação teve um crescimento de produtividade de 2,95\%, ao ano, entre 1990 e 1998.

O cálculo da produtividade no setor serviços podem ser visto no Gráfico 4. Nele, observamos que a produtividade dos serviços cresceu entre 1990 e 1994, com tendência de queda a partir de 1995, apesar das oscilações.

Gráfico 4: Produtividade e variações percentuais acumuladas do pessoal ocupado e valor adicionado do setor serviços, exceto setor público - 1990 - 2006

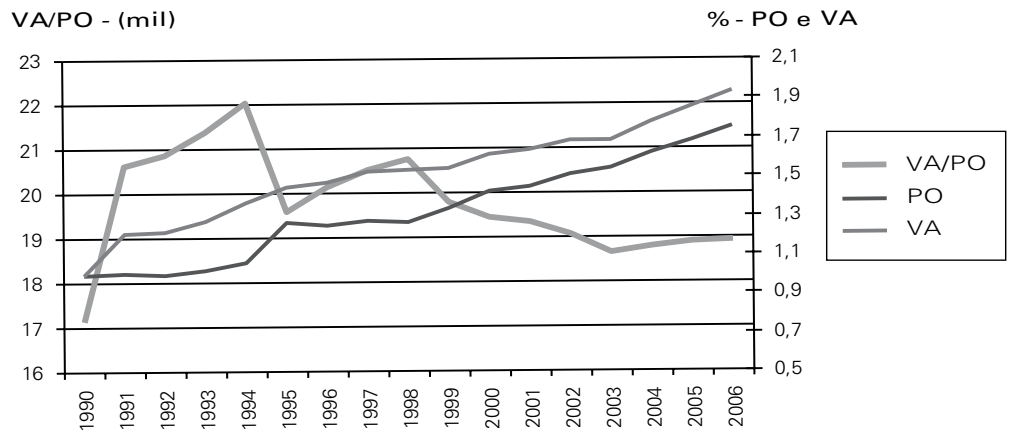

Fonte: Elaboração própria com dados do Sistema de Contas Nacionais - IBGE.

\footnotetext{
${ }^{5}$ Rossi Jr. e Ferreira (1999): aumento médio anual de 6,21\% entre 1990 e 1993 e 7,97\% entre 1994 e 1997; Feijó Carvalho e Almeida (2005): aumento médio anual de 2,3\% entre 1990 e 2003; Carvalheiro (2003): aumento médio anual entre 1990 e 2000 de 1,14\%; e Ferraz, Kupfer e Iootty (2004): uma duplicação da relação produto-trabalho entre 1990 e 1999.
} 
Assim, a evolução da produtividade do trabalho nos dois setores apresenta algumas semelhanças importantes: enquanto a produtividade em ambos apresentou um crescimento médio anual de $2,7 \%$ para os serviços e $2,95 \%$ para a indústria de transformação, entre 1990 e 1998; após esse período até 2006, essas taxas foram de $-1,1 \%$ e $-1,55 \%$, respectivamente. De acordo com os resultados apresentados acima, é de esperar um aumento na demanda relativa por mão de obra com maior qualificação nos dois setores apenas até 1998, supondo que a maior produtividade tenha sido acompanhada de complementaridade entre investimento e trabalho qualificado e/ou que os novos processos de produção aumentaram a demanda por trabalhadores qualificados.

\section{OFERTA E DEMANDA POR QUALIFICAÇÃO NO BRASIL}

\section{Relação entre nível de emprego e nível do salário real por escolaridade segundo setores}

Para analisar a evolução da demanda por qualificação na economia brasileira, foram extraídos os dados do número de empregados desagregados por faixas de escolaridade e por setor de atividade, segundo Setores de Atividade Econômica da Relação Anual de Informações Sociais (RAIS) ${ }^{6}$. As séries foram divididas por setores da economia brasileira: serviços incluindo o comércio sem administração pública, serviços exceto comércio e administração pública, indústria de transformação e agropecuária ${ }^{7}$.

As faixas de escolaridade são aquelas disponíveis na RAIS: analfabeto; da $1^{\text {a }}$ a $5^{a}$ série de fundamental incompleta; $5^{a}$ série do fundamental completa; $6^{a}$ a $9^{a}$ série do fundamental incompleta; fundamental completo; médio incompleto; médio completo; superior incompleto; superior completo. As faixas mestrado, doutorado e ignorados não são analisadas por apresentarem pequena participação e não serem computadas em todos os anos pesquisados.

As séries abrangem o período 1990-2006, o que rendeu uma amostra média de cerca 15 milhões de empregados por ano. O Gráfico 5 apresenta os resultados em milhões de trabalhadores. Nele, podemos observar que, nos serviços e na indús-

\footnotetext{
${ }^{6}$ A RAIS tem uma importante limitação quanto aos dados que disponibiliza. Somente os trabalhadores formalmente empregados estão registrados nela. Trabalhadores autônomos, domésticos e informais não são computados na mesma, o que pode gerar viés de seleção na amostra utilizada. Em que pese suas limitações, a descontinuidade e as mudanças metodológicas frequentes em outras bases de dados bastante utilizadas gerariam resultados distorcidos ou que não contemplariam todo o período que se deseja analisar.

${ }^{7}$ A indústria extrativa mineral e a construção, bem como os serviços industriais de utilidade pública atendem a dinâmicas diferentes e respondem por parcelas reduzidas da força de trabalho no Brasil. Por estes motivos a análise se concentra na indústria de transformação. Cabe ressaltar que o setor público é o maior empregador de mão de obra com ensino superior no Brasil, mas como a dinâmica de sua demanda difere daquela da economia privada ele não foi incluído na análise.
} 
tria de transformação, o número de empregos nas três faixas de menor escolarização caiu, em termos absolutos. A agropecuária apresentou resultados inexpressivos, não tendo alterado significativamente a composição do emprego segundo escolarização da sua força de trabalho.

Ainda pelos dados apresentados no Gráfico 5, notamos que as faixas etárias " $6^{a}$ a $9^{a}$ série do fundamental" sofreram poucas alterações em todos os setores analisados. Por outro lado, ocorreu aumento generalizado no número de empregados com maior escolaridade, sobretudo daqueles com ensino médio completo. De acordo com a parte superior do Gráfico 5, notamos que o comércio absorveu boa parte da mão de obra com ensino médio completo. Para os níveis "superior incompleto" e "superior completo", o crescimento foi significativo no setor de serviços, mas não na indústria de transformação.

Gráfico 5: Evolução da quantidade de empregos em quatro setores da economia brasileira segundo graus de escolaridade - 1990- 2006
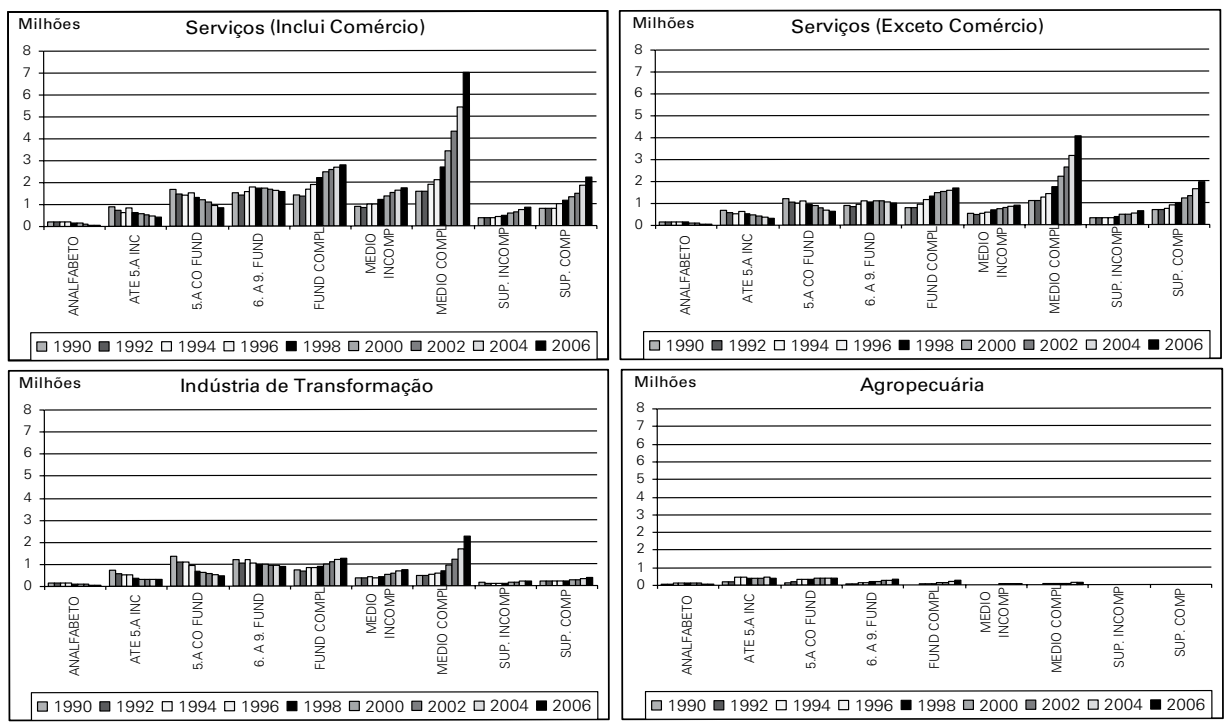

Fonte: Elaboração própria a partir de dados da RAIS.

Nota: Os painéis foram colocados em uma mesma escala para facilitar a comparação entre os setores.

Estes resultados estão de acordo com aqueles encontrados por Fernandes e Menezes-Filho (2002), em que os autores verificaram uma substituição de trabalhadores não qualificados por trabalhadores com qualificação intermediária na economia brasileira, na década de 1990. O dados apresentados no Gráfico 5 sugerem que esse fenômeno se intensificou a partir de 2000 .

O crescimento no número de postos de trabalho para pessoas com o ensino médio completo é o que mais se destaca na análise dos painéis acima. Entre 1990 e 2006, dos empregos líquidos gerados, somando-se os setores selecionados, $89 \%$ estavam associados a pessoas com este grau de escolaridade. 
No mesmo período ocorreu um aumento no número de declarações de empregados com ensino superior completo da ordem de 1,594 milhão. O setor de serviços novamente se destaca, com elevação de 1,41 milhão. A indústria de transformação foi a que apresentou os resultados menos expressivos, criando apenas 166 mil empregos líquidos para trabalhadores com esse nível de ensino ${ }^{8}$.

O período entre 1990 e 1998 foi aquele cujas produtividades da indústria e dos serviços mais cresceram e, portanto, período em que se esperava aumento na contratação de trabalhadores qualificados, principalmente por parte da indústria de transformação. Entretanto, esta registrou demissões líquidas de 1.379 empregados graduados, no período. Já o setor de serviços, incluindo comércio, apresentou contratações líquidas de 357.017 desses profissionais. Contudo, o fato da indústria de transformação não ter apresentado contratações líquidas no período não significa, necessariamente, que a demanda relativa por mão de obra com ensino superior tenha caído no segmento, pois ocorreu uma retração na mão de obra total empregada neste, de acordo com os dados apresentados no Gráfico 2.

O Gráfico 6 fornece uma ideia melhor da participação relativa dos empregados em cada segmento de acordo com o nível de qualificação. Nele, podemos verificar que a relevância, em todos os setores, do crescimento relativo do emprego de trabalhadores com ensino médio completo. Entre 1990 e 1998, a indústria de transformação e os serviços, incluindo comércio, apresentaram aumento nas participações destes de $6,12 \%$ e $5,27 \%$ para $24,87 \%$ e $23,28 \%$, respectivamente.

Em relação ao ensino superior completo, a indústria apresentou aumento de $0,88 \%$ e os serviços, incluindo o comércio, de $1,54 \%$. Sem o comércio esse valor vai para $2,19 \%$. Assim, o setor de serviços não apresentou suficiente elevação de mão de obra com ensino superior completo para justificar um processo relevante de fluxo de trabalho da indústria de transformação para o setor de serviços devido a um processo de terceirização de alguns serviços que eram realizados anteriormente pela própria indústria, pelo menos para trabalhadores com esse nível de qualificação.

\footnotetext{
${ }^{8}$ Em 1990, do total de empregados com ensino superior completo, 31,33\% estavam nos serviços, $10,11 \%$ na indústria de transformação e $3,76 \%$ no comércio. Em 2006, estes números foram para $37,29 \%, 7,57 \%$ e $5,12 \%$, respectivamente.
} 
Gráfico 6: Evolução do percentual de empregados em três setores da economia brasileira segundo graus de escolaridade - 1990- 2006
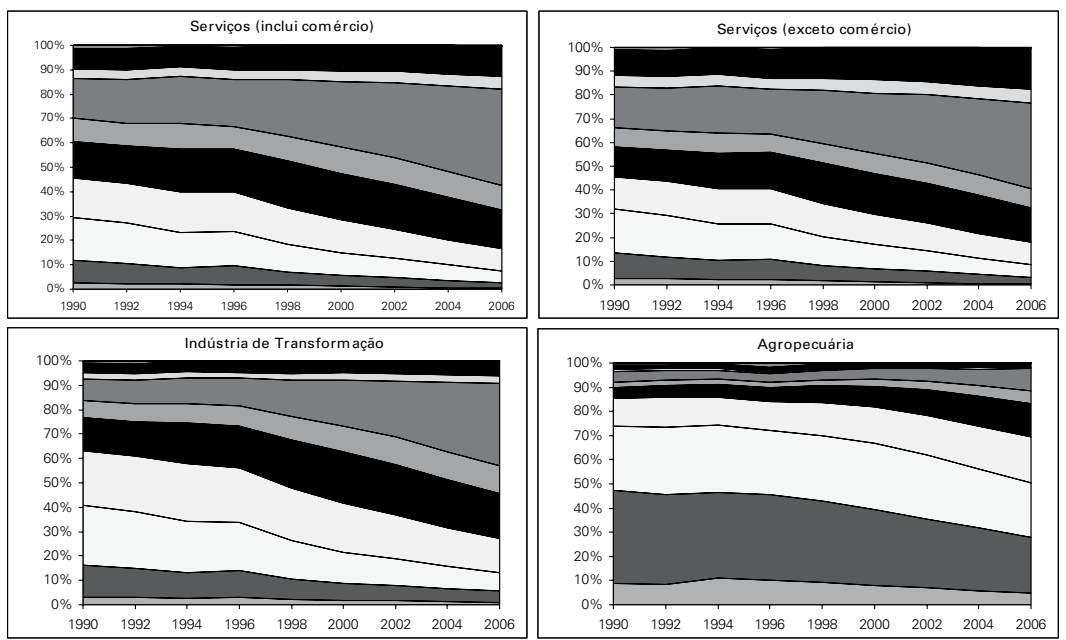

Fonte: Elaboração própria a partir de dados da RAIS.

Nota: Os painéis foram colocados em uma mesma escala para facilitar a comparação entre os setores.

A partir deste ponto, duas hipóteses se configuram. A primeira é a de que esses trabalhadores com qualificação intermediária (ensino médio completo) passaram a ser, efetivamente, mais demandados pelos setores produtivos devido às mudanças estruturais ocorridas na economia brasileira. A segunda é a de que esse aumento teria respondido a mudanças nas condições de oferta de pessoas com ensino médio completo, em função dos esforços governamentais em promover aumentos na escolaridade da população, intensificados desde a década de 1990.

Para se ter uma percepção mais acurada deste processo, analisamos a evolução salarial real por setores de atividade desagregados segundo escolaridade dos empregados ${ }^{9}$. Os resultados estão expostos no Gráfico 7. Por meio deste, podemos constatar, de maneira geral, que os salários dos trabalhadores com menos escolaridade (até o ensino fundamental completo) apresentaram poucas variações entre 1990 e 2006. Para as faixas intermediárias (do ensino médio incompleto ao superior incompleto) ocorreram quedas nos salários reais entre 1990 e 2006, embora com um pequeno acréscimo entre 1990 e 1998.

\footnotetext{
${ }^{9}$ Para isso, utilizamos os dados da RAIS. O MTE disponibiliza os salários mínimos médios mensais por setor de atividade a cada ano. Estes, se multiplicados pelos salários mínimos reais médios mensais dos anos correspondentes, fornecem valores aproximados dos salários reais médios mensais para cada ano. Os dados do salário mínimo médio mensal foram retirados do IPEADATA, que os disponibiliza em Reais do último mês da série (agosto de 2008, no presente estudo). Foi estabelecida a seguinte equação para o cálculo dos salários reais médios por setor de atividade para cada grau de escolaridade em um determinado ano: $\bar{S}_{j i t}=\bar{R}_{j i t}\left(\frac{1}{12} \sum_{m=1}^{12} s_{m i}\right)$ onde $\bar{S}_{i j t}$ corresponde ao salário real médio no setor $i$, na escolaridade $j$, no ano $t ; \bar{R}_{i j t}$ corresponde à remuneração média mensal no setor $i$, na escolaridade $j$, no ano $t$, em salários mínimos; e $s_{m_{t}}$ corresponde ao salário mínimo médio mensal real no setor $m$ do ano $t$.
} 
Os salários dos trabalhadores com nível superior apresentaram elevações mais expressivas entre 1990 e 1998, em todos os setores (até 1996 para a agropecuária). A partir de então, os salários sofreram uma reversão, embora uma parte do crescimento inicial tenha se mantido até o final da série, principalmente no caso da indústria de transformação.

Gráfico 7: Salário real médio em quatro setores da economia brasileira segundo graus de escolaridade- (r\$ de agosto de 2008) - 1990 - 2006
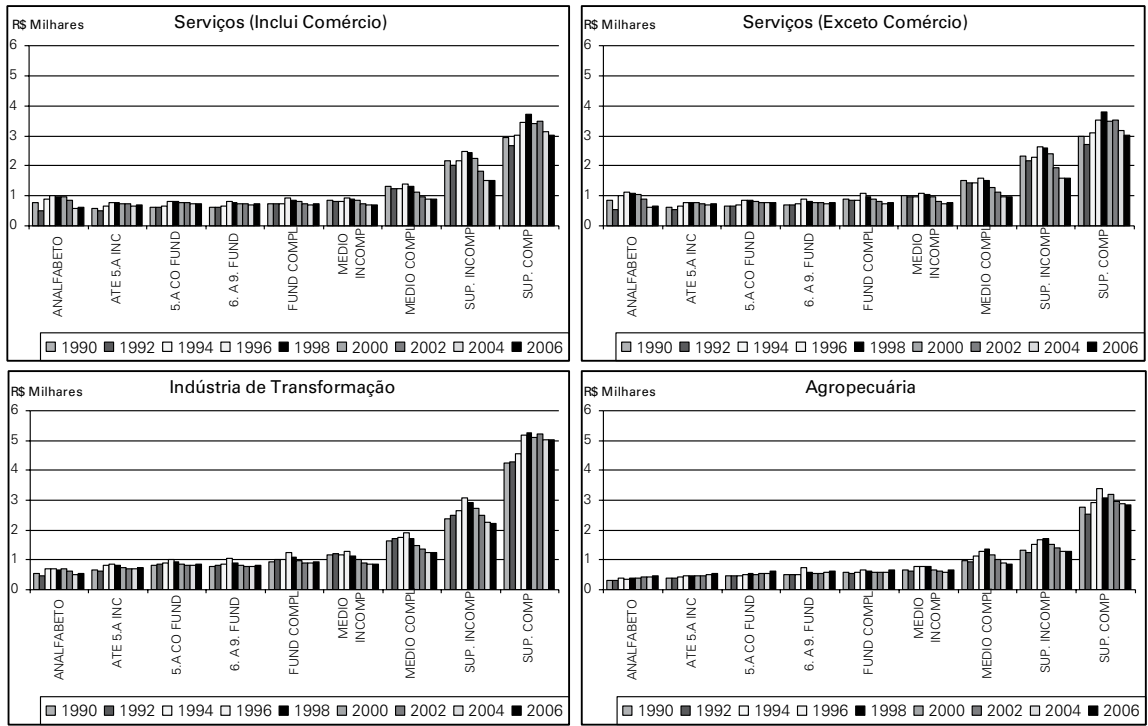

Fonte: Elaboração própria a partir de dados da RAIS e IPEADATA (salário mínimo real).

Ainda pelo Gráfico 7, podemos constatar que os salários dos empregados com ensino superior completo da indústria de transformação, dos serviços exceto comércio e dos serviços em geral apresentaram aumentos de $18,42 \%, 1,71 \%$ e $2,92 \%$, respectivamente, entre 1990 e 2006. Apesar da grande elevação do emprego de mão de obra com ensino superior no setor de serviços, o aumento dos salários foi maior na indústria de transformação. Isso é uma evidência que os trabalhadores com esse nível de qualificação que não encontraram emprego neste migraram para aquele. Desse modo, os aumentos salariais na indústria apontam que a demanda por qualificação neste setor aumentou.

Por outro lado, os trabalhadores com nível de ensino médio incompleto, médio completo e superior incompleto experimentaram reduções nos níveis reais de salário tanto na indústria quanto nos serviços ${ }^{10}$.

\footnotetext{
${ }^{10}$ No setor de serviços, os trabalhadores com nível de ensino médio incompleto, médio completo e superior incompleto experimentaram reduções salariais de $16,81 \%, 33,45 \%$ e $30,51 \%$, entre 1990 e 2006 , respectivamente. Para a indústria de transformação, as reduções foram de $25,43 \%, 25,01 \%$ e $7,95 \%$, respectivamente.
} 
De acordo com os dados apresentados no Gráfico 7, os grupos de escolaridade que registraram os maiores aumentos no número de trabalhadores (do fundamental completo ao médio completo), também registraram as mais significativas reduções salariais. Assim, podemos concluir que os empregos associados aos níveis de escolaridade médios apresentaram aumento da oferta superior ao aumento da demanda, pressionando os salários para baixo, o que está de acordo com os resultados apresentados por Andrade e Menezes-Filho (2005). Esses efeitos ficam ainda mais evidentes pela análise de decomposição realizada na próxima subseção.

\section{Análise de decomposição da escolaridade e dos salários}

Outra maneira de analisarmos a demanda por trabalho qualificado no Brasil é verificar se a ampliação no nível de escolaridade (salário) dos trabalhadores foram ou não acompanhados por mudanças em suas ocupações utilizando o método da decomposição ${ }^{11}$. Esse método consiste em verificar se os aumentos de escolaridade estão associados a deslocamentos dos empregados entre as ocupações ou internamente a elas ${ }^{12}$.

Em relação à escolaridade, a variável cujas oscilações foram decompostas foi o grau de instrução médio, por setores e para toda a amostra. A decomposição da escolaridade dos trabalhadores foi realizada de acordo com a seguinte equação:

$$
\frac{\Delta I M}{I M_{0}}=\frac{\sum_{i=1}^{3540 u 584}\left(I M_{i 0} \times \Delta P_{i}\right)}{I M_{0}}+\frac{\sum_{i=1}^{3540 u 584}\left(P_{i o} \times \Delta I M_{i}\right)}{I M_{0}}+\frac{\sum_{i=1}^{3540 u 584}\left(\Delta P_{i} \times \Delta I M_{i}\right)}{I M_{0}}
$$

onde IM é o grau de instrução médio, $i$ é o índice que se refere a $i$-ésima ocupação, $P$ é a participação da ocupação no emprego total (total da amostra obtida na RAIS $)^{13}$. Em relação aos salários, a variável cujas oscilações foram decompostas foi o salário real médio por setor e, posteriormente, para toda a amostra. Os cálculos da decomposição dos salários foram realizados de acordo com:

$$
\frac{\Delta S M}{S M_{0}}=\frac{\sum_{i=1}^{354 \text { ou } 584}\left(S M_{i 0} \times \Delta P_{i}\right)}{S M_{0}}+\frac{\sum_{i=1}^{354 \text { ou } 584}\left(P_{i o} \times \Delta S M_{i}\right)}{S M_{0}}+\frac{\sum_{i=1}^{354 \text { ou } 584}\left(\Delta P_{i} \times \Delta S M_{i}\right)}{S M_{0}}
$$

\footnotetext{
${ }^{11}$ Por exemplo, Kruger (2008), Porcile et al. (2008), Fagerberg (2000), Bernard e Jones (1995) e Carvalheiro (2003).

${ }^{12}$ A decomposição foi baseada em Fagerberg (2000), Carvalheiro (2003) e Holland e Porcile (2005).

${ }^{13}$ O sobrescrito do sinal de somatório contém as quantidades de ocupações da CBO 1994 (354 ocupações) e CBO 2002 (584 ocupações), cabendo usar a quantidade correspondente de acordo com a classificação utilizada.
} 
onde $S M$ é o salário real médio, $i$ é o índice que se refere a $i$-ésima ocupação e $P$ é a participação da ocupação no emprego total.

Cada termo das decomposições acima captura uma determinada fonte de variação na variável que se deseja analisar. O primeiro termo, denominado "estático", captura a variação na participação das diferentes ocupações no emprego total, mantendo a escolaridade (salário) fixa no ano base. Em outras palavras, ele captura o efeito de uma variação média da escolaridade (salário) devido a uma alteração da participação das diferentes ocupações no emprego total, pois o nível médio de escolaridade (salário) é distinto em cada uma delas.

O segundo termo, denominado "interno", captura o efeito da variação da escolaridade (salário) considerando que a participação de cada ocupação no emprego total permaneça constante. Desse modo, esse termo quantifica a elevação da escolaridade (salário) em cada ocupação internamente, ou seja, considerando a mesma estrutura produtiva em termos de ocupação.

Finalmente, o terceiro termo, denominado "dinâmico", mede a interação entre mudanças na escolaridade (salário) em cada segmento e a alocação do trabalho entre eles. Esse termo será positivo se as ocupações com maior crescimento relativo do emprego forem aquelas em que seus trabalhadores experimentaram ganhos de escolaridade (salário), considerando o período em questão.

Foram realizadas decomposições separadamente para cada setor segundo classificação do IBGE (Setores de Atividade Econômica) e para a economia como um todo, utilizando os dados da RAIS. Para a variável ocupação, tanto na decomposição da escolaridade como na salarial, foi utilizada a Classificação Brasileira de Ocupações CBO com desagregação de três dígitos (grupo base de ocupação: 354 ocupações, CBO-1994) para os anos de 1985, 1990, 1994, 1998 e 2002 e desagregação de 4 dígitos (família ocupacional: 584 ocupações, CBO-2002) para os anos de 2003 e 2007.

Devido ao fato de 17,5\% das ocupações listadas na CBO-2002 não apresentar correspondência com a CBO-1994, optou-se por não decompor o período completo, mas apenas seus subperíodos. Com isso, a variação tanto da escolaridade média, quanto dos salários médios, entre os anos de 2002 e 2003, também não puderam ser decompostas. Assim, as decomposições foram realizadas para os seguintes subperíodos: 1985-1990, 1990-1994, 1994-1998, 1998-2002 e 2003-2007.

Para a variável escolaridade, foi necessário estipular quantos anos de estudo uma determinada faixa disponível na RAIS corresponderia. Para aquelas faixas onde um determinado grau de escolaridade não estava completo foi utilizada a média dos anos de estudo das faixas anterior e posterior. Os resultados foram os seguintes: analfabeto $=0$; até o $5^{\mathrm{a}}$ ano incompleto do ensino fundamental $=2,5 ; 5^{\mathrm{a}}$ ano completo do ensino fundamental $=5$; Do $5^{\text {a }}$ ao $9^{\mathrm{a}}$ ano incompleto do ensino fundamental $=7$; ensino fundamental completo $=9$; ensino médio incompleto $=$ 10,5 ; ensino médio completo $=12$; educação superior incompleta $=14$; educação superior completa $=16$; mestrado completo $=18$ e doutorado completo $=22^{14}$.

$\mathrm{Na}$ análise salarial, a variável "remuneração em dezembro em salários mínimos"

\footnotetext{
${ }^{14}$ Os níveis mestrado e doutorado foram incluídos aqui, mas, da mesma maneira que na análise acima, suas contribuições para os resultados alcançados são inexpressivas.
} 
da RAIS foi utilizada para toda a série, em valores reais. Optou-se por esta classificação, pois ela está disponível em todos os anos pesquisados, o que não ocorre com os dados dos salários em Reais ${ }^{15}$. Outra característica relevante é que a escolaridade dos empregados na RAIS é referente ao mês de dezembro. Os resultados estão apresentados na Tabela 1.

Tabela 1: Decomposição das variações educacionais e salariais no Brasil para setores selecionados - 1985 - 2007

\begin{tabular}{|c|c|c|c|c|c|c|c|c|c|}
\hline \multirow{2}{*}{ Setor } & \multirow{2}{*}{ Período } & \multicolumn{4}{|c|}{ Decomposição Educacional (\%) } & \multicolumn{4}{|c|}{ Decomposição Salarial (\%) } \\
\hline & & Estático & Dinâmico & Interno & Total & Estático & Dinâmico & Interno & Total \\
\hline \multirow{5}{*}{ Ind. Transf. } & $1985-1990$ & 0,86 & 0,08 & 6,84 & 7,79 & 2,43 & $-0,86$ & $-20,24$ & $-18,67$ \\
\hline & 1990-1994 & $-1,38$ & 0,32 & 5,85 & 4,79 & $-1,33$ & 0,97 & 33,57 & 33,21 \\
\hline & 1994-1998 & 0,05 & $-0,06$ & 8,33 & 8,33 & $-1,90$ & 0,62 & 4,43 & 3,15 \\
\hline & 1998-2002 & $-0,77$ & 0,07 & 9,73 & 9,03 & $-2,12$ & 0,45 & $-11,01$ & $-12,68$ \\
\hline & 2003-2007 & $-0,19$ & 0,13 & 7,24 & 7,19 & 0,45 & $-0,21$ & 4,26 & 4,50 \\
\hline \multirow{5}{*}{ Serviços } & $1985-1990$ & $-1,85$ & 0,80 & 3,88 & 2,83 & $-0,94$ & $-0,13$ & $-10,80$ & $-11,87$ \\
\hline & 1990-1994 & $-0,43$ & 0,02 & 4,27 & 3,86 & $-0,56$ & 1,83 & 23,16 & 24,43 \\
\hline & 1994-1998 & $-0,04$ & 0,18 & 5,21 & 5,35 & $-4,58$ & 1,50 & 23,55 & 20,47 \\
\hline & 1998-2002 & $-0,17$ & 0,04 & 7,35 & 7,23 & $-0,89$ & 0,90 & $-22,02$ & $-22,01$ \\
\hline & 2003-2007 & 1,11 & $-0,17$ & 5,37 & 6,31 & 0,52 & $-0,36$ & 3,46 & 3,62 \\
\hline \multirow{5}{*}{ Comércio } & 1985-1990 & $-0,43$ & $-0,21$ & 18,01 & 17,36 & 1,36 & $-0,58$ & $-23,78$ & $-23,00$ \\
\hline & 1990-1994 & 0,51 & 0,13 & $-7,14$ & $-6,50$ & 0,32 & 0,80 & 26,28 & 27,39 \\
\hline & 1994-1998 & $-0,65$ & 0,15 & 4,68 & 4,19 & $-1,31$ & $-0,82$ & 11,43 & 9,30 \\
\hline & 1998-2002 & $-0,11$ & 0,02 & 8,24 & 8,15 & 0,02 & $-0,19$ & $-12,01$ & $-12,18$ \\
\hline & 2003-2007 & 0,30 & $-0,07$ & 5,65 & 5,88 & 0,83 & $-0,38$ & 10,58 & 11,03 \\
\hline \multirow{5}{*}{$\begin{array}{l}\text { Agrope- } \\
\text { cuária }\end{array}$} & 1985-1990 & 4,64 & 0,09 & 7,63 & 12,35 & 3,32 & $-1,44$ & $-28,36$ & $-26,47$ \\
\hline & 1990-1994 & $-6,97$ & $-1,43$ & 5,89 & $-2,51$ & $-7,49$ & $-0,18$ & 29,24 & 21,57 \\
\hline & 1994-1998 & $-0,85$ & 0,01 & 6,23 & 5,39 & $-4,75$ & 1,09 & 16,12 & 12,46 \\
\hline & 1998-2002 & $-0,64$ & 0,44 & 11,21 & 11,00 & $-2,72$ & 0,43 & $-7,87$ & $-10,16$ \\
\hline & 2003-2007 & 1,49 & $-0,07$ & 12,99 & 14,42 & 1,97 & $-0,21$ & 17,50 & 19,26 \\
\hline \multirow{5}{*}{$\begin{array}{l}\text { Total da } \\
\text { economia }\end{array}$} & 1985-1990 & 0,44 & 0,03 & 2,39 & 2,87 & 0,93 & $-0,38$ & $-15,45$ & $-14,90$ \\
\hline & 1990-1994 & $-0,49$ & $-0,07$ & 4,31 & 3,75 & $-1,13$ & $-0,99$ & 27,06 & 24,93 \\
\hline & 1994-1998 & $-0,06$ & $-0,02$ & 5,12 & 5,04 & $-2,44$ & 0,48 & 18,17 & 16,21 \\
\hline & 1998-2002 & 0,02 & $-0,17$ & 8,01 & 7,86 & $-1,13$ & $-0,67$ & $-11,47$ & $-13,27$ \\
\hline & 2003-2007 & 0,22 & $-0,02$ & 5,56 & 5,77 & 0,59 & $-1,04$ & 10,16 & 9,71 \\
\hline
\end{tabular}

Fonte: Elaboração própria com dados da RAIS. Nota: Serviços exclui o comércio e o setor público.

${ }^{15}$ A conversão de salários mínimos para salários em Reais foi realizada multiplicando-se o salário mínimo médio de uma determinada ocupação, em dezembro, pelo valor do salário mínimo real do mesmo mês, em reais de dezembro de 2008, de acordo com os dados do Salário Mínimo Real, do Instituto de Pesquisa Econômica Aplicada (IPEA). Com isso, a série foi automaticamente deflacionada. 
De acordo com os resultados dessa tabela, o termo estático da análise da decomposição foi negativo ou muito pequeno em praticamente todos os períodos e segmentos, entre 1990 e 2002, para as duas variáveis analisadas. Esse comportamento indica que o fraco desempenho econômico brasileiro nos anos 1990 foi acompanhado por uma mudança estrutural desfavorável tanto à melhora da qualificação do trabalhador quanto à elevação do nível salarial. Com a melhora do desempenho econômico, a partir de 2003, a mudança estrutural ocorreu no sentido de elevar a escolaridade dos trabalhadores e o nível salarial, com exceção da indústria de transformação para a escolaridade. Ou seja, mesmo com a recuperação econômica, a mudança estrutural que ocorreu nesta não foi no sentido de elevar a demanda por trabalhadores qualificados.

Adicionalmente, observa-se que a variação interna da escolaridade e dos salários predominou (isto é, foi maior em módulo, sendo mais representativa da variação total) em todos os períodos analisados e todos os setores, com exceção da agropecuária entre 1990 e 1994, onde o termo estático apresentou maior peso na variação total.

Em relação à componente estática (ou termo estático) da decomposição da escolaridade, observa-se que não há um padrão único entre os setores e os valores oscilam entre positivos e negativos, com frequência. Além disso, esses valores são bastante reduzidos, revelando que não ocorreram oscilações relevantes da escolaridade dos trabalhadores brasileiros provenientes de mudanças na estrutura das ocupações.

A indústria de transformação apresenta valores negativos para essa componente em três dos cinco subperíodos analisados. Assim, nesses subperíodos, as ocupações que apresentavam maiores escolaridades médias foram as que menos cresceram em termos de participação no emprego. Ou seja, as alterações na demanda, com impactos na estrutura da economia agiram negativamente na escolaridade média da indústria de transformação.

A componente dinâmica, por sua vez, é a que apresenta a menor contribuição para o aumento da escolaridade dos setores e para a economia como um todo. Esse fato indica que aqueles setores que mais ganharam participação em termos de número de empregados não foram os mesmos que apresentaram ganhos na escolaridade dos trabalhadores.

O reduzido efeito das componentes estática e dinâmica, mesmo com o processo de abertura comercial e redução significativa da participação do emprego industrial no total da economia brasileira reflete, em parte, certa estabilidade em sua estrutura produtiva. Ferraz, Kupfer e Iootty (2004) mostram que, entre 1990 e 2002, apesar das grandes transformações ocorridas na indústria em relação à produtividade do trabalho, poucas atividades econômicas foram adicionadas à matriz industrial brasileira, assim como poucas deixaram de existir. Pelos resultados apresentados no exercício de decomposição, esse processo foi semelhante entre 2003 e $2007^{16}$.

Em suma, grande parte do aumento da escolaridade se deu internamente às ocupações. Como já dito, uma explicação possível para esse fenômeno é a do aumento da qualificação dos trabalhadores devido a programas governamentais. Ou-

\footnotetext{
${ }^{16}$ Não aprofundamos essa análise da variação da participação dos diferentes segmentos da indústria e dos demais setores da economia brasileira no período, pois fugiria do objetivo do presente estudo.
} 
tra possibilidade é que esse aumento seja, em parte, uma resposta a maior demanda por qualificação dos empregados em função da difusão da informática em grande parte das ocupações. Isto é, poucas ocupações teriam ficado imunes às necessidades de capacitação inerentes à difusão da utilização da informática, o que pressionou o aumento da escolaridade dos trabalhadores sem que ocorressem alterações em suas ocupações.

Para saber qual dessas duas hipóteses é a mais provável, precisamos analisar o comportamento dos salários. O aumento da oferta de trabalhadores qualificados devido a programas governamentais que criam um descompasso entre oferta e demanda de trabalhadores deveria ter impactos negativos sobre os salários. Já o aumento da demanda por qualificação pelo efeito da difusão da informática ou outros fatores que elevam a demanda deveria resultar em maiores salários. Para verificar essas duas possibilidades, as correlações entre as componentes das decomposições educacionais e salariais foram calculadas para todo o período da análise ${ }^{17}$.

Tabela 2: Correlação entre as componentes das decomposições

\begin{tabular}{|l|c|c|c|c|}
\hline \multicolumn{1}{|c|}{ Setor } & Estático & Dinâmico & Interno & Total \\
\hline Ind. Transform. & 0,72 & 0,26 & $-0,54$ & $-0,86$ \\
Serviços & 0,14 & $-0,24$ & $-0,50$ & $-0,29$ \\
Comércio & 0,39 & 0,34 & $-0,93$ & $-0,94$ \\
Agropecuária & 0,93 & 0,11 & $-0,13$ & $-0,50$ \\
Total dos quatro & 0,79 & 0,14 & $-0,47$ & $-0,58$ \\
\hline
\end{tabular}

Fonte: Elaboração própria com dados da RAIS.

Observa-se que a componente interna apresentou sinal negativo em todos os setores. Isso significa que nos períodos em que os aumentos da escolaridade foram maiores os aumentos salariais reais foram menores ou negativos. Ou seja, esses resultados evidenciam que um aumento da escolaridade dentro da mesma ocupação foi, em geral, acompanhado por uma queda nos salários. Assim, os fatores de oferta foram mais relevantes para explicar as mudanças ocorridas no nível de escolaridade dos trabalhadores das diferentes ocupações.

Pela magnitude dos coeficientes apresentados na Tabela 2, o segmento de comércio foi o que apresentou esse efeito de forma mais acentuada. Ou seja, os resultados indicam que esse foi o segmento que mais absorveu mão de obra com maior qualificação sem que ocorressem alterações em sua demanda.

Esse padrão de qualificação dos trabalhadores nos leva a algumas indagações. Primeiramente, uma elevação na qualificação da oferta de mão de obra sem um acompanhamento da demanda, com consequente redução dos salários reais pode ser um indício de que os recursos com educação não estão sendo bem gastos. Em segundo lugar, pode ser que a elevação no nível de escolaridade dos trabalhadores

\footnotetext{
${ }^{17}$ Por exemplo, os valores do componente estático educacional da indústria de transformação foram correlacionados com os valores do componente estático salarial do mesmo setor. Isso foi repetido cada setor e para os quatro setores em conjunto, para cada termo da decomposição.
} 
ocorreu de forma puramente quantitativa, ou seja, com pouco reflexo de sua qualificação. O fato de que uma grande parte dessa absorção de mão de obra com maior qualificação, principalmente daqueles trabalhadores com ensino médio completo, ocorreu justamente em um segmento que em que as atividades não são complexas - comércio - e que, consequentemente, não exige trabalhadores com elevado nível de qualificação indica que o segundo fator é relevante para explicar a evolução dessas variáveis.

Em relação às correlações dos componentes estático educacional e estático salarial da análise de decomposição, os resultados mostram que os valores são positivos para todos os setores (Tabela 2). Desse modo, os efeitos das mudanças estruturais sobre a qualificação dos trabalhadores ocorreram na mesma direção dos seus efeitos sobre os salários. Adicionalmente, de acordo com os resultados apresentados na Tabela 1, verificamos que o componente estático contribuiu de forma negativa para a elevação da escolaridade média dos trabalhadores, se bem que de forma marginal. Interpretando os dois resultados de forma conjunta, concluímos que a mudança estrutural nas ocupações levou a uma redução na escolaridade dos trabalhadores e, consequentemente, uma redução em seus respectivos salários.

Em resumo, o que se percebe é que essa mudança estrutural gerou uma redução marginal na demanda por trabalhadores qualificados. Ou seja, a mudança estrutural pela qual o país vem passando desde o início da década de 1990 não tem atuado de modo a gerar um maior dinamismo econômico. Em um país com reduzido nível de escolaridade, a ocorrência de mudanças na estrutura que não incentivem a qualificação dos trabalhadores não pode ser positiva se, de fato, essa variável é relevante no bem-estar social, crescimento e distribuição da renda. Por outro lado, a elevação da escolaridade da mão de obra vem ocorrendo via políticas públicas com esse objetivo sem, no entanto, dinamizar a economia.

Resultados similares de que a mudança estrutural nas últimas décadas não foi benéfica para o dinamismo da economia brasileira foram encontrados por cruz et al. (2007). No entanto, estes focaram nos efeitos da mudança estrutural sobre a participação dos setores de acordo com seus respectivos níveis de tecnologia, enquanto, no presente estudo, analisamos seus impactos na demanda por trabalho de acordo com seu nível de qualificação.

\section{CONCLUSÃO}

As evoluções do produto e do emprego na economia brasileira apresentadas no presente estudo mostram que ocorreram mudanças estruturais significativas na economia do país entre 1990 e 1999, além de elevação significativa na produtividade, principalmente da indústria de transformação. A partir do ano 2000, houve relativa estabilidade nestas variáveis, quando se realiza a análise dos dados der forma agregada.

No entanto, a demanda por trabalhadores qualificados não aumentou nos períodos em que ocorreram os maiores ganhos de produtividade em termos relativos e absolutos na indústria de transformação e no setor de serviços.

Houve, por outro lado, aumentos expressivos nas quantidades de trabalhado- 
res com qualificação intermediária (ensino fundamental ou médio completos) em todos os setores. Além disso, os salários dos mesmos caíram de forma acentuada no período de maior expansão da oferta de trabalhadores com esse nível de qualificação, ou seja, entre 1998 e 2006.

Em geral, as correlações negativas dos componentes salarial e educacional internos mostram que o aumento da quantidade de trabalhadores esteve associado a quedas nos salários médios em todos os setores, principalmente no comércio. Adicionalmente, as correlações positivas dos componentes estático educacional e estático salarial mostram que os efeitos das mudanças estruturais ocorreram de forma a reduzir a escolaridade média dos trabalhadores, além de seus salários.

Esses resultados indicam, em primeiro lugar, que os aumentos da escolaridade média dos trabalhadores brasileiros têm respondido, em maior medida, à expansão da oferta de ensino no país devido a programas governamentais. Em segundo lugar, as mudanças estruturais ocorridas, principalmente na década de 1990, não têm agido de modo a aumentar a demanda por esse contingente crescente de trabalhadores com maior qualificação. Por fim, os aumentos de produtividade observados na década de 1990 não foram acompanhados por aumentos na quantidade de trabalhadores qualificados nos serviços e na indústria de transformação.

Pode-se, assim, dizer que a mudança estrutural pela qual o país vem passando a partir do início dos anos 1990 não é benéfica no sentido de elevar a demanda por mão de obra qualificada e por gerar um dinamismo que promova o crescimento econômico. Adicionalmente, os resultados indicam que a elevação no nível de escolaridade por si só não traz efeitos relevantes sobre a dinâmica do mercado. Antes de tudo, é preciso que sejam tomadas medidas para que setores dinâmicos e que demandam mão de obra qualificada sejam estimulados.

Outra possibilidade é que a elevação na escolaridade dos trabalhadores não ocorreu de forma a melhorar o nível de habilidade dos mesmos. Ou seja, houve uma elevação na escolaridade média dos trabalhadores com queda na qualidade do ensino. Nesse caso, seria necessário estimular incrementos na qualidade do ensino para que se possa produzir um dinamismo que promova a elevação da produtividade e crescimento sustentável do produto dos diferentes setores. No entanto, uma análise mais aprofundada para verificar quais dos dois efeitos acima citados é o mais relevante para explicar o comportamento dos salários dos trabalhadores dos setores formais da economia brasileira está fora do objetivo do presente estudo e deverá ser abordado em análises futuras.

\section{REFERÊNCIAS BIBLIOGRÁFICAS}

ANDRADE, A. A. S.; MENEZES-FILHO, N. A. (2005). O papel da oferta de trabalho no comportamento dos retornos à educação no Brasil. Pesquisa e Planejamento Econômico, v.35, n.2.

BERNARD, A.B.; JONES, C.I. (1995). Comparing apples to oranges: productivity convergence and measurement across industries and countries. American Economic Review 86 (5), 1216-1238.

BONELLI, R.; FONSECA, R. (1998). Ganhos de produtividade e de eficiência: novos resultados para a economia brasileira. Pesquisa e Planejamento Econômico, v. 28, n. 2, p. 273-314. Rio de Janeiro: Ipea. 
BRESSER-PEREIRA, L.C.; NAKANO, Y. (2003). Crescimento Econômico com Poupança Externa? Revista de Economia Política, 23 (2): 3-27.

CARVALHEIRO, N. (2003). Uma decomposição do aumento da produtividade do trabalho no brasil durante os anos 90. Revista de Economia Contemporânea. Rio de Janeiro, 7(1): 81-109, jan./jun.

CARVALHO, P. G. M.; FEIJÓ, C. A. (2000). Produtividade industrial no Brasil: o debate recente. Indicadores Econômicos FEE, 28, 3, pp. 631-646.

CRUZ, M.J.V.; NAKABASHI, L.; PORCILE, G.; SCATOLIN, F. D. (2007). Uma análise do impacto da composição ocupacional sobre o crescimento da economia brasileira. Economia, Revista da Anpec, 08 (Selecta): 55-73.

DASGUPTA, S.; SINGH, A. (2006). Manufacturing, Services and Premature Deindustrialization in Developing Countries, a Kaldorian Analysis. United Nations University Research Paper, 2006/49: 1-19.

DASGUPTA, S.; SINGH, A. (2005). Will Services Be the New Engine of Indian Economic Growth? Development and Change, 36 (06): 1035-58.

FAGERBERG, J. (2000). Technological progress, structural change and productivity growth: a comparative study. Structural Change and Economic Dynamics 11: 393-411.

FEIJÓ, C. A.; CARVALHO, P. G. M.; ALMEIDA, J. S. G. (2005). Ocorreu uma desendustrialização no Brasil? Instituto de Estudos para o Desenvolvimento Industrial. Mimeo.

FERNANDES, R.; MENEZES-FILHO, N. A. (2002). Escolaridade e Demanda Relativa por Trabalho: Uma Avaliação para o Brasil nas Décadas de 80 e 90 . Mimeo.

FERRAZ, J. C.; KUPFER, D.; IOOTTY, M. (2004). Industrial Competitiveness in Brazil: Ten Years After Economic Liberalization. CEPAL Review, (82): 92-117.

HOLLAND, M.; PORCILE, G. (2005). Brecha tecnológica y crecimiento en América Latina. In Cimoli, M., editor, Heterogeneidad Estructural, Asimetrías Tecnológicas y Crecimiento en América Latina. BID-CEPAL, Santiago.

KONGSAMUT, P.; REBELO, S.; XIE, D. (2001). Beyond balanced growth. Reviewof Economic Studies 68: 869-882.

KRÜGER, J.J. (2008). Productivity and structural change: a review of the literature. Journal of Economic Surveys. Vol. 22, No. 2, pp. 330-363

MACHADO, A. F.; MOREIR A, M. M. (2001). Os impactos da abertura comercial sobre a remuneração relativa do trabalho no Brasil. Belo Horizonte: Faculdade de Ciências Econômicas, Universidade Federal de Minas Gerais.

MADDISON, A. (1995). Monitoring the World Economy. 1820-1992. OECD, Paris.

MENEZES-FILHO, N. A.; RODRIGUES JR., M. (2003). Tecnologia e Demanda por Qualificação na Indústria Brasileira. Revista Brasileira de Economia. Rio de Janeiro 57(3):569-603.

NASSIF, A. (2006). Há evidências de desindustrialização no Brasil? Texto para Discussão do Banco Nacional de Desenvolvimento Econômico e Social (BNDES), 108.

PALMA, J. G. (2005). Four sources of deindustrialization and a new concept of the Dutch disease. In: OCAMPO, J.A. (ed.). Beyond reforms. Palo Alto (CA): Stanford University Press.

PORCILE, G.; CRUZ, M. J. V.; NAKABASHI, L.; SCATOLIN, F. D. (2008). Structural change and the service sector in Brazil. Mimeo.

ROSSI JR., J. L.; FERREIRA, P. C. (1999). Evolução da produtividade industrial brasileira e abertura comercial. Rio de Janeiro: IPEA, Texto para Discussão n. 651.

ROWTHORN. R., (1999). Indústria de transformação: crescimento, comércio e mudança estrutural. In: O futuro da indústria no Brasil e no Mundo, os desafios do século XXI (1999): 57-76. Rio de Janeiro: Editora Campus.

ROWTHORN, R.; RAMASWAMY, R. (1999). Growth, Trade and Deindustrialization. In: IMF Staff Papers, vol. 46, no. 1., pp. 18-41.

SALM, C.; SABOIA, J.; CARVALHO, P. (1997). Produtividade na indústria brasileira: questões metodológicas e novas evidências empíricas. Pesquisa e Planejamento Econômico. Rio de Janeiro: Ipea.

SASAKI, H. (2007). The rise of service employment and its impact on aggregate productivity growth. Structural Change and Economic Dynamics. 18 (2007) 438-459. 